\title{
Quality of life in patients with gastric cancer: translation and psychometric evaluation of the Iranian version of EORTC QLQ-STO22
}

\author{
Sanamber Sadighi*1, Ali Montazeri*2,3, Zahra Sedighi ${ }^{1}$, \\ Mohammad Ali Mohagheghi ${ }^{1}$ and Hossein Froutan ${ }^{4}$
}

Address: ${ }^{1}$ Cancer Research Center, Cancer Institute, Tehran University of Medical Sciences, Tehran, Iran, ${ }^{2}$ Department of Mental Health, Iranian Institute for Health Sciences Research, ACECR, Tehran, Iran, ${ }^{3}$ Public Health and Health Policy, Division of Community-Based Sciences, Faculty of Medicine, University of Glasgow, Glasgow, UK and ${ }^{4}$ Department of Gastroentrology, Tehran University of Medical Sciences, Tehran, Iran

Email: Sanamber Sadighi* - ssadighi@doctor.com; Ali Montazeri* - montazeri@acecr.ac.ir; Zahra Sedighi - sedighi_zahra@yahoo.com; Mohammad Ali Mohagheghi - mamohagheghi@yahoo.com; Hossein Froutan - froutan@ams.ac.ir

* Corresponding authors

Published: 28 August 2009

BMC Cancer 2009, 9:305 doi:10.1 186/147|-2407-9-305
Received: 27 April 2009

Accepted: 28 August 2009

This article is available from: http://www.biomedcentral.com//47I-2407/9/305

(C) 2009 Sadighi et al; licensee BioMed Central Ltd.

This is an Open Access article distributed under the terms of the Creative Commons Attribution License (http://creativecommons.org/licenses/by/2.0), which permits unrestricted use, distribution, and reproduction in any medium, provided the original work is properly cited.

\begin{abstract}
Background: Disease and treatment related events, can adversely affect the quality of life of patients with cancer. The purpose of this study was to translate and validate a gastric cancer specific health related quality of life questionnaire (EORTC QLQ-STO22) for Iranian patients suffering from gastric cancer.

Methods: Forward-backward procedure was applied to translate the English language version of the EORTC QLQ-STO22 into Persian (Iranian language). Then, the questionnaire and the EORTC core quality of life instrument (QLQ-C30) were administered to a sample of patients with confirmed diagnosis of gastric cancer. All patients filled in questionnaires before and after one month of treatment. Patients were divided into two groups based on intension of treatment (curative vs. palliative). Reliability and validity of the module was tested by internal consistency and known group comparisons, respectively.

Results: In all, 105 patients were entered into the study. Cronbach's alpha for multi-item scales (to test reliability) ranged from 0.54 to 0.87 . The questionnaire discriminated well between clinically distinct subgroups of patients both before and after treatment lending support to its convergent and clinical validity.

Conclusion: Overall, the Iranian version of the EORTC QLQ-STO22 demonstrated a good reliability and clinical validity to support its use in combination with core questionnaire in outcome studies of gastric cancer in Iran. However, using the QLQ-STO22 in a wide range of Iranian patients with gastric cancer should allow further confirmation for its psychometric properties.
\end{abstract}

\section{Background}

Although mortality rates due to gastric cancer had been declining for several decades, on a worldwide scale its incidence is still high, and it is the second leading cause of cancer death, behind lung cancer $[1,2]$. Recent efforts to improve survival include pre and post-operative chemo- 
therapy and chemo-radiotherapy. However, improvements in survival with multi-modal treatment may also be associated with increased toxic side effects. Therefore full evaluation of new treatments of gastric cancer should be included patient-reported outcome measures such as health related quality of life (HRQOL) as well as assessment of biomarkers, pathologic responses or survival outcomes.

Although quality of life in patients with gastric cancer is increasingly added as outcome measure in clinical research, it is argued that quality of life assessments in these patients deserve more systematic studies using gastric cancer specific instruments. A recent review of the literature on quality of life in gastric cancer indicated that in most reported studies quality of life was assessed mainly with generic measures, and the social dimensions of quality of life were largely neglected [3]. In assessing quality of life in cancer patients it is recommended that to use a cancer-specific questionnaire as a general measure of quality of life in cancer patients (e.g. the EORTC QLQ-C30) plus site-specific modules (e.g. breast cancer specific or gastric cancer specific). Thus, for instance the EORTC, in addition to core cancer quality of life questionnaire, has developed several site-specific questionnaires including gastric specific quality of life measures (the EORTC QLQ-STO22) in order to collect more relevant patient-reported outcomes in studying quality of life in this group of cancer patients.

The QLQ-STO22 has been translated into many languages such Dutch, Danish, French, German, Geek, Hungarian, Italian, Japanese, Korea, Norwegian, Portuguese, Brazilian, Russian, Spanish, Taiwanese and Turkish $[4,5]$.

Gastric cancer is the most common cancer in Iran and according to the latest published data there are more than 5000 new cases and equally about 5000 deaths each year due to gastric cancer [6]. Thus as one might realize studying quality of life in gastric cancer patients in Iran is very important and relevant. Since the EORTC QLQ-STO22 was not available in Iran, this study carried out to translate and provide evidence for its psychometric properties in Iran so that the questionnaire could be used in the future outcome studies in gastric cancer patients with the hope that this might contribute to the existing literature and help to improve quality of life among these cancer patients.

\section{Methods}

\section{Design and data collection}

This was an observational 4-week follow up study conducted in the Medical Oncology Department of the Cancer Research Center of the Tehran University of Medical Science. A consecutive sample of patients were entered into the study during March 2005 to September 2007. Eli- gible cases were gastric cancer patients with confirmed diagnosis and life expectancy of at least 4 weeks. Patients were excluded if they had concurrent malignancies or if they were unable to understand the questionnaire.

\section{The questionnaire}

Permission was asked from the EORTC Quality of Life Department to develop the Iranian version of the EORTC gastric cancer specific quality of life questionnaire (EORTC QLQ-STO22). We used the standard 'forwardbackward' procedure in order to translate the English language version of the EORTC QLQ-STO22 into Persian (Iranian language). The translated module was reviewed, pre-tested, revised and its final form was used in this study. In addition the Iranian version of the EORTC QLQC30 was administered to patients. The psychometric properties of the Iranian version of the EORTC QLQ-C30 are well documented [7]. Patients completed the questionnaires before starting chemotherapy or supportive care. The second assessment was carried out four weeks later. At baseline assessment, patients were asked to complete a short debriefing questionnaire about the time took to complete the questionnaires, the need for help in completing the questionnaire and indicating if any of the items appeared confusing, difficult to answer or upsetting. Demographic and treatment data were also recorded.

The EORTC QLQ-STO22 module contains 22 items in a similar layout and response format to the EORTC QLQC30. The module consists of five multi-item scales (dysphagia, eating restrictions, pain, reflux, and anxiety) and four single items (dry mouth, body image, hair loss, and taste problem). Higher scores on the QLQ-STO22 represent greater level of symptoms [5].

\section{Statistical analysis}

Reliability: internal consistency and test-retest analyses were performed to test reliability. The internal consistency of the multi-item scales was assessed by Cronbach's alpha coefficient at baseline and four week later. Values equal to or greater than 0.7 were considered satisfactory [8]. Testretest reliability of the questionnaire was examined using intraclass correlation coefficients (ICC) between pre- and post-treatment assessments. Values of ICC vary from zero (totally unreliable) to 1 (perfectly reliable). Values above 0.80 were considered as evidence of excellent reliability [9].

Validity: convergent validity and clinical validity were performed to examine scale validity. Convergent validity for each scale was assessed using the correlation between each item and its own scale corrected for overlap. It was expected that the correlation between an item and its own scale was significantly higher than its correlation with other scales. Pearson's correlation coefficient was used to 
test convergent validity and values of 0.40 or above were considered satisfactory ( $\mathrm{r} \geq 0.81-1.0$ as excellent, 0.61 0.80 very good, $0.41-0.60$ good, $0.21-0.40$ fair, and $0-0.20$ poor) $[8,9]$.

Known group comparisons was used for analysis of the clinical validity of the Iranian version of the QLQ-STO22 in order to explore the extent to which the questionnaire is able to discriminate between subgroups of patients. Known groups used for this comparison were treatment groups (potentially curative vs. palliative). Group differences were assessed using a non-parametric test (MannWhitney U test).

\section{Ethics}

Ethics committee of Tehran University of Medical Sciences approved the study. Written informed consent was obtained from all enrolled patients.

\section{Results}

In total, 105 patients filled in both questionnaires the EORTC QLQ-C30 and QLQ-STO22. Of these, 50 had loco-regional disease and received curative therapy (chemotherapy and surgery), while 55 had advanced disease and poor performance and received palliative treatment. The mean age of patients was 58.1 years $(\mathrm{SD}=$ $10.7)$, and $76 \%$ were male $(n=72)$. The average time required to complete the questionnaire was 5 minutes. Almost all patients found the questionnaire easy to understand and acceptable. However, a few patients commented that they could not differentiate between 'acid indigestion and hurt burn' (item 39) or some patients stated that they could not understand what does 'trouble with belching' really mean while in general belching is unpleasant condition (item 40).
Table 1 shows the internal consistency for the five QLQSTO22 multi-item scales. In general all scales except eating restrictions $(\alpha=0.54)$ showed satisfactory results. Intraclass correlation coefficient (ICC) values for the Iranian version of QLQ-STO22 also indicated acceptable test-retest reliability for the questionnaire. ICC values ranged from 0.53 for eating restrictions to 0.84 for dysphagia.

Item convergent validity of the QLQ-STO22 is shown in Table 2. There were a desirable correlation between each item and its own scale lending support to its item-component validity. As indicated in Table 2 the correlation between an item and its own scale was significantly higher than its correlation with other scales.

Table 3 and Table 4 are presenting the results obtained from known group comparisons before and after treatment. In both assessments, patients in different groups that are curative and palliative treatment groups showed significant differences for most quality of life scores.

\section{Discussion}

This was a validation study of the EORTC QLO-STO22 in Iran and in general the questionnaire showed promising psychometric results. In addition patients received it well and we did not notice any problems when it was administered to the Iranian patients reflecting the fact that the translation was satisfactory and easy to understand.

In general the reliability of the Iranian version of the QLQ-STO22 was relatively good. However, internal consistency for two multi-item scales (reflux and eating restrictions) was lower than recommended value. The internal consistency for reflux subscale at baseline assessment was 0.62 and for eating restrictions at both assess-

Table I: Descriptive statistics and scale reliability of the EORTC QLQ-STO22 $(n=105)$

\begin{tabular}{lllll}
\hline & Pre treatment & Follow up & Mean (SD) & Cronhbac's alpha* \\
\hline Multi-item Scales** & & Cronhbach's alpha* & & \\
\hline Dysphagia & $21.8(25.0)$ & 0.72 & $20.0(26.3)$ & 0.81 \\
\hline Stomach pain & $33.1(26.3)$ & 0.78 & $30.1(26.3)$ & 0.87 \\
\hline Reflux & $26.8(23.1)$ & 0.62 & $27.2(24.5)$ & 0.74 \\
\hline Eating restrictions & $32.8(14.0)$ & 0.54 & $31.1(15.2)$ & 0.61 \\
\hline Anxiety & $56(31.3)$ & 0.87 & $55.8(32.8)$ & 0.93 \\
\hline
\end{tabular}

* A value of 0.70 or above indicates adequate reliability

** Scores range from 0 to 100 (higher scores indicate worse conditions) 
Table 2: Correlation between multi-item scales and its own scale corrected for overlap (figures are Pearson correlation coefficient obtained from pretreatment assessment)*

\begin{tabular}{|c|c|c|c|c|c|}
\hline & Dysphagia & Pain & Reflux & Eating restrictions & Anxiety \\
\hline \multicolumn{6}{|l|}{ Dysphagia } \\
\hline Problems eating solid foods & 0.82 & 0.52 & 0.40 & 0.22 & 0.21 \\
\hline Problems eating liquidized or soft food & 0.87 & 0.34 & 0.29 & 0.01 & 0.08 \\
\hline Problems drinking liquids & 0.78 & 0.17 & 0.23 & 0.04 & 0.03 \\
\hline \multicolumn{6}{|l|}{ Pain } \\
\hline Eating discomfort & 0.61 & 0.76 & 0.55 & 0.32 & 0.33 \\
\hline Stomach pain & 0.31 & 0.84 & 0.38 & 0.34 & 0.23 \\
\hline Stomach discomfort & 0.27 & 0.89 & 0.46 & 0.42 & 0.31 \\
\hline Abdomen bolting & 0.21 & 0.62 & 0.41 & 0.24 & 0.36 \\
\hline \multicolumn{6}{|l|}{ Reflux } \\
\hline Acid or bile problem & 0.30 & 0.44 & 0.83 & 0.21 & 0.30 \\
\hline Acid indigestion or heartburn & 0.24 & 0.52 & 0.73 & 0.21 & 0.18 \\
\hline Belching trouble & 0.35 & 0.33 & 0.69 & 0.39 & 0.25 \\
\hline \multicolumn{6}{|l|}{ Eating restrictions } \\
\hline Full up too quickly & 0.34 & 0.54 & 0.46 & 0.74 & 0.35 \\
\hline Trouble enjoying meals & 0.38 & 0.34 & 0.20 & 0.72 & 0.08 \\
\hline Long time to complete meals & 0.20 & 0.40 & 0.33 & 0.74 & 0.38 \\
\hline Eating trouble in front of other people & 0.08 & 0.46 & 0.35 & 0.77 & 0.20 \\
\hline
\end{tabular}

\section{Anxiety}

\begin{tabular}{lccccc}
\hline Thinking about illness & 0.19 & 0.39 & 0.31 & 0.43 & $\mathbf{0 . 9 2}$ \\
\hline Worry about low weight & 0.18 & 0.42 & 0.37 & 0.34 & $\mathbf{0 . 8 5}$ \\
\hline Health worry & 0.04 & 0.28 & 0.01 & 0.07 & $\mathbf{0 . 4 6}$ \\
\hline
\end{tabular}

* Similar results were obtained from the post-treatment assessment. To avoid confusion the correlation coefficients are not presented in this table.

** Excellent correlation $\geq 0.81-1.0,0.61-0.80$ very good, $0.41-0.60$ good, $0.21-0.40$ fair, and $0-0.20$ poor

ments was 0.54 , and 0.61 respectively. It seems that since the internal consistency for the reflux improved in the second assessment thus it could be regarded as satisfactory. But it appears that the low internal consistency for the eating restrictions subscale could not be neglected. It seems that item 42 (have you had trouble enjoying your meals?) that belongs to this subscale needs some modification in the future studies. Item 42 is a very straightforward question and we did not have nor any problems in translating the item into Persian language nor any patients indicated difficulties in responding to this question. Yet, we are not certain why the internal consistency for this scale was lower than recommended value. Perhaps 'enjoyment of meals' might mean different thing in our culture as com- 
Table 3: The known groups comparison before treatment

\begin{tabular}{|c|c|c|c|}
\hline & $\begin{array}{l}\text { Curative treatment } \\
(n=50)\end{array}$ & $\begin{array}{l}\text { Palliative treatment } \\
(n=55)\end{array}$ & \\
\hline & Mean (SD) & Mean (SD) & $\mathbf{P} *$ \\
\hline \multicolumn{4}{|l|}{ EORTC QLQ-C30 } \\
\hline \multicolumn{4}{|l|}{ Functioning scores** } \\
\hline Physical functioning & $75.3(15.8)$ & $55.1(23.5)$ & $<0.0001$ \\
\hline Role functioning & (19.5) 77.7 & $60.3(27.3)$ & 0.001 \\
\hline Emotional functioning & $67.1(25.1)$ & $53.4(26.6)$ & 0.008 \\
\hline Cognitive functioning & $96.4(10.9)$ & $90.6(14.7)$ & 0.009 \\
\hline Social functioning & $78.6(16.7)$ & $(25.2) 71.8$ & 0.26 \\
\hline Global quality of life & $45.7(11.8)$ & $39.2(10.5)$ & 0.007 \\
\hline \multicolumn{4}{|l|}{ Symptom scores*** } \\
\hline Fatigue & $36.0(24.4)$ & $56.8(27.1)$ & $<0.0001$ \\
\hline Nausea \& vomiting & $10.0(22.3)$ & $24.2(28.1)$ & 0.001 \\
\hline Pain & $30.4(24.1)$ & $52.8(28.3)$ & $<0.0001$ \\
\hline Dyspnea & $6.0(14.5)$ & $9.6(20.9)$ & 0.51 \\
\hline Insomnia & $27.3(33.4)$ & $46.0(35.4)$ & 0.006 \\
\hline Appetite loss & $34.0(38.9)$ & $54.0(37.6)$ & 0.007 \\
\hline Constipation & $10.6(24.6)$ & $27.7(37.6)$ & 0.009 \\
\hline Diahrea & $5.3(15.5)$ & $5.5(16.8)$ & 0.91 \\
\hline Financial difficulties & $80.8(30.0)$ & $72.8(36.6)$ & 0.26 \\
\hline \multicolumn{4}{|c|}{ QLQ-STO22 scores**** } \\
\hline Dysphagia & $15.6(20.6)$ & $29.1(28.3)$ & 0.01 \\
\hline Stomach area pain & $25.0(24.4)$ & $38.3(24.4)$ & 0.005 \\
\hline Reflux & $20.3(20.1)$ & $30.3(24.6)$ & 0.03 \\
\hline Eating restrictions & $29.9(14.9)$ & $33.3(16.1)$ & 0.44 \\
\hline Anxiety & $50.3(27.6)$ & $65.3(29.4)$ & 0.01 \\
\hline
\end{tabular}


Table 3: The known groups comparison before treatment (Continued)

\begin{tabular}{lccc}
\hline Dry mouth & $29.2(35.1)$ & $52.3(35.3)$ & 0.001 \\
\hline Taste problem & $20.4(32.5)$ & $31.2(33.6)$ & 0.06 \\
\hline Body image & $20.9(24.7)$ & $26.6(33.5)$ & 0.73 \\
\hline Hair loss & $17.3(33.4)$ & $18.8(27.2)$ & 0.51 \\
\hline
\end{tabular}

* $\mathrm{P}$ values derived from Mann-Whitney test

** Scores range from 0 to 100 with higher scores indicating better conditions.

*** Scores range from 0 to 100 with higher scores indicating greater level of symptoms.

Table 4: The know groups comparison after treatment

\begin{tabular}{|c|c|c|c|}
\hline & $\begin{array}{l}\text { Curative treatment } \\
(n=50)\end{array}$ & $\begin{array}{l}\text { Palliative treatment } \\
(n=55)\end{array}$ & \\
\hline & Mean (SD) & Mean (SD) & $\mathbf{P} *$ \\
\hline \multicolumn{4}{|l|}{ EORTC QLQ-C30 } \\
\hline \multicolumn{4}{|l|}{ Functioning scores** } \\
\hline Physical functioning & $71.4(21.8)$ & $52.7(23.6)$ & $<0.0001$ \\
\hline Role functioning & $74.4(22.6)$ & $61.2(27.4)$ & 0.02 \\
\hline Emotional functioning & $66.2(26.1)$ & $56.9(25.5)$ & 0.08 \\
\hline Cognitive functioning & $94.4(12.8)$ & $89.1(17.7)$ & 0.07 \\
\hline Social functioning & $79.4(16.1)$ & $68.9(26.8)$ & 0.12 \\
\hline Global quality of life & $50.1(18.2)$ & 38.4 (11.9) & 0.003 \\
\hline \multicolumn{4}{|l|}{ Symptom scores*** } \\
\hline Fatigue & $40.0(26.1)$ & $54.3(29.9)$ & 0.01 \\
\hline Nausea \& vomiting & $15.9(19.8)$ & $33.3(32.7)$ & 0.01 \\
\hline Pain & $25.9(22.0)$ & $43.3(28.0)$ & 0.001 \\
\hline Dyspnea & $2.8(9.4)$ & $12.0(20.1)$ & 0.009 \\
\hline Insomnia & $29.7(31.6)$ & $42.7(35.5)$ & 0.07 \\
\hline Appetite loss & $10.6(24.6)$ & $27.7(37.6)$ & 0.009 \\
\hline Constipation & $10.8(23.3)$ & 19.1 (27.5) & 0.05 \\
\hline Diahrea & $11.5(21.3)$ & $15.6(28.5)$ & 0.63 \\
\hline Financial difficulties & $78.0(32.1)$ & $77.7(33.3)$ & 0.89 \\
\hline
\end{tabular}

QLQ-STO22 scores*** 
Table 4: The know groups comparison after treatment (Continued)

\begin{tabular}{|c|c|c|c|}
\hline Dysphagia & $14.0(23.0)$ & $27.2(28.6)$ & 0.009 \\
\hline Stomach area pain & I8.I (19.3) & $43.6(26.3)$ & $<0.0001$ \\
\hline Reflux & $20.2(21.8)$ & $36.3(24.5)$ & 0.001 \\
\hline Eating restrictions & $29.9(15.1)$ & $34.3(15.4)$ & 0.09 \\
\hline Anxiety & $46.0(34.7)$ & $58.8(32.3)$ & 0.15 \\
\hline Dry mouth & $38.5(36.8)$ & $53.6(36.4)$ & 0.05 \\
\hline Taste problem & $24.0(30.2)$ & $35.8(34.9)$ & 0.11 \\
\hline Body image & $25.0(35.7)$ & $38.3(37.8)$ & 0.25 \\
\hline Hair loss & $39.6(33.1)$ & $51.4(34.6)$ & 0.14 \\
\hline
\end{tabular}

* $\mathrm{P}$ values derived from Mann-Whitney test

** Scores range from 0 to 100 with higher scores indicating better conditions.

*** Scores range from 0 to 100 with higher scores indicating greater level of symptoms.

pared to the meaning of 'enjoyment of meals' in western countries. It is not surprising that a similar study from Taiwan also reported that the internal consistency of the eating restrictions subscale was lower that recommended value (0.67) [10]. In addition, although a study from Japan reported high internal consistency for all five multiitem subscales including eating restrictions subscale (ranging from 0.76 to 0.88 ), it was found that item 42 showed higher correlation with dysphagia scale and even in factor analysis loaded highly on pain subscale rather that eating restrictions subscale. As suggested there might be a need to discuss with EORTC Quality of Life Group to establish an agreement on the various language versions of the QLQ-STO22 [11].

Clinical validity of the questionnaire, as examined by using known-group comparisons, showed satisfactory results. The questionnaire discriminated well between two groups that differed in their clinical status receiving two different regimens. Differences in quality of life scores on both measures (QLQ-C30 and QLQ-STO22) between patients who received curative treatment and those who received palliative treatment in most instances were significant at pre- and post-treatment assessments (Table 3 and Table 4). However, unlike most studies already cited in this paper there were no significant differences between two groups for social functioning. One possible explanation for such observation might be related to the fact that in Iran social ties are relatively very strong and thus both patient groups received equal support from family, relatives and friends.
The present study indicated that global quality of life was the most adversely affected subscale among the respondents while others have shown that patients scored lower on social functioning [12]. Lower score for global quality of life in other cancer patients were also reported. For instance, a recent study form Kuwait (very similar in culture to our patients) reported that global quality of life in breast cancer was lower than other functioning scores [13]. Such similarities or dissimilarities between patients from different cultures might worth to be studied further. However, it seems that effective treatment could help to improve quality of life in gastric cancer patients $[14,15]$.

Although there was excellent patient compliance, we need more exploration of cross-cultural differences. Further studies with larger samples are also needed to confirm the sensitivity to changes over time.

\section{Conclusion}

Overall the Iranian version of the EORTC QLQ-STO22 showed that it is a reliable and valid specific measure of quality of life in patients with gastric cancer. However, using the QLQ-STO22 in a wide range of Iranian patients with gastric cancer should allow further confirmation for its psychometric properties.

\section{Competing interests}

The authors declare that they have no competing interests.

\section{Authors' contributions}

SS and AM were the main investigators. SS wrote the first draft and designed the study. AM analyzed the data and wrote the final manuscript. ZS contributed to the data col- 
lection and data entry. MAM and HF contributed to the study design and patient management. All authors read and approved the final manuscript.

\section{Acknowledgements}

The authors wish to thank all colleagues who helped to carry out this study. The research deputy of Tehran University of Medical Sciences supported this study.

\section{References}

I. Smith RP, Shinohara E: Gastric cancer: The Basics. [http:// www.oncolink.com/types/article. $\mathrm{cfm} ? \mathrm{c}=5 \& \mathrm{~s}=\mid 4 \& \mathrm{ss}=105 \&$ id $=9463]$. [accessed 26 April 2009]

2. Bertuccio P, Chatenoud L, Levi F, Praud D, Ferlay J, Negri E, Malvezzi M, La Vecchia C: Recent patterns in gastric cancer: A global overview. Int J Cancer 2009, I 25:666-673.

3. Kaptein AA, Morita S, Sakomato J: Quality of life in gastric cancer. World J Gastroenterol 2005, II:3189-3196.

4. Vickery CW, Blazeby JM, Conroy T, Arraras J, Sezer O, Moore J, Koller, Rosemeyer D, Johnson CD, Alderson D: Development of an EORTC disease-specific quality of life module for use in patients with gastric Cancer. Eur J Cancer 200I, 37:966-97I.

5. Blazeby JM, Conroy T, Bottomley A, Vickery C, Arraras J, Sezer O, Moore J, Koller M, Turhal NS, Stuart R, van Cutsem E, D'haese S, Coens $C$ : Clinical and psychometric validation of a questionnaire module, the EORTC QLQ-STO 22, to assess quality of life in patients with gastric cancer. Eur J Cancer 2004, 40:2260-2268.

6. Mousavi SM, Gouya MM, Ramazani M, Davanlou N, Hajsadeghi N, Sedighi Z: Cancer incidence and mortality in Iran. Ann Oncol 2009, 20:556-563.

7. Montazeri A, Harirchi I, Vahdani M, Khaleghi F, Jarvandi S, Ebrahimi M, Haji-Mahmoodi M: The European Organization for Research and Treatment of Cancer Quality of Life Questionnaire (EORTC QLQ-C30): translation and validation study of the Iranian version. Supportive Care Cancer 1999, 7:400-406.

8. Nunnally JC, Bernstein IR: Psychometric theory. New York: McGraw- Hill; 1994.

9. Norman GR, Streiner DL: Biostatistics: The bare essentials. St. Louis, MO: Mosby; 1994.

10. Huang CC, Lien HH, Sung YC, Liu HT, Chie WC: Quality of life of patients with gastric cancer in Taiwan: validation and clinical application of the Taiwan Chinese version of the EORTC QLQ-C30 and EORTC QLQ-STO22. Psycho-Oncology 2007, 16:945-949.

11. Morita S, Kaptein AA, Oba K, Sakomoto J: The domain structure of the EORTC QLQ-STO22 supported by Japanese validation data. Psycho-Oncology 2008, I7:474-479.

12. Kim WS, Park YH, Lee SH, Ryoo BY, Yang SH, Lee SS, Kim MS, Kim K, Park KW, Im do H, Kang JH, lee J, Ko YH, ahn YC, Lom do H, Park K, Bang SM: Quality of life one year after chemoradiotherapy for localized primary gastric diffuse large B-cell lymphoma. Med Oncol 2008, 25:447-450.

13. Alawadi SA, Ohaeri JU: Health-related quality of life of Kuwaiti women with breast cancer: a comparative study using the EORTC Quality of Life Questionnaire. BMC Cancer 2009, 9:222.

14. Sadighi S, Mohagheghi MA, Montazeri A, Sedighi Z: Quality of life in Patients with advanced gastric cancer: a randomized trial comparing TCF with ECF. BMC Cancer 2006, 6:274.

15. Bae JM, kun S, Kim YW, Ryu KW, Lee JH, Noh JH, Sohn TS, Hong SK, Park SM, You CH, Kim JH, Lee MK, Yun YH: Health-related quality of life among disease-free stomach cancer survivors in Korea. Qual Life Res 2006, I5:1587-1596.

\section{Pre-publication history}

The pre-publication history for this paper can be accessed here:

http://www.biomedcentral.com/1471-2407/9/305/pre pub
Publish with Biomed Central and every scientist can read your work free of charge

"BioMed Central will be the most significant development for disseminating the results of biomedical research in our lifetime. "

Sir Paul Nurse, Cancer Research UK

Your research papers will be:

- available free of charge to the entire biomedical community

- peer reviewed and published immediately upon acceptance

- cited in PubMed and archived on PubMed Central

- yours - you keep the copyright
BiolMedcentral 